\title{
Trigonometria Indiana: o método das diferenças, as séries de potências do seno, cosseno, e as estimativas de $\pi$.
}

Marcos Rodrigues (D) Nilton Barroso (1)

\section{Resumo}

No presente trabalho apresentamos um resumo da trigonometria indiana a partir do trabalho de Ariabata I e seu método de construção de tabela de seno. Com esse contexto, matemáticos indianos posteriores demonstraram como calcular seno e cosseno usando série de potência sem a utilização do Cálculo de Newton e Leibniz. Por último, descrevemos o método indiano para o cálculo de $\pi$ através de uma série numérica associada à função arctan.

Palavras-chave: Matemática indiana; série de potência; seno; cosseno; pi.

\section{Abstract}

In the following work we present a summary of Indian trignometry based on the work of Ariabata I and his method of building a sine table. In this context, later Indian mathematicians demonstrated how to calculate sine and cosine using a power series without using Newton and Leibniz Calculus. Finally, we describe the Indian method to calculate $\pi$ using a numerical series associated with the arctan function.

Keywords: Indian mathematics; power series; sine; cosine; pi.

\section{Introdução}

Os primeiros registros da matemática indiana são encontrados em textos religiosos da Era Védica (c. 500 a.C.) que continham manuais para construção de altares em formas geométricas diversas. Como no caso das civilizações da Mesopotâmia e egípcia, no início a matemática na Índia tinha um caráter iminentemente prático, e era utilizada como ferramenta indispensável para solução dos mais diversos problemas relevantes ao dia a dia das grandes cidades que começavam a surgir: elaboração de calendários precisos necessários para a agricultura e celebração de datas religiosas, construção de edificações, divisão da terra etc. Nos mil anos que se seguiram, mesmo após sucessivas alternâncias de poder, surpreendentemente a produção matemática indiana não foi abandonada. Pelo contrário, foi estimulada e passou por uma significativa transformação conceitual, adotando uma abordagem mais intuitiva e abstrata. 
O período clássico da matemática indiana inicia-se na geração de Ariabata I [Āryabhața] ${ }^{1}$ (c. 500 d.C) e Brahmagupta [Brahmaguptah] (c. 600 d.C.). Seus trabalhos foram ao mesmo tempo um resumo da matemática conhecida anteriormente e o início de uma nova era para essa disciplina. São eles os responsáveis por abandonar contextos religiosos para desenvolver técnicas de astronomia e trigonometria modernas [2], com destaque para a utilização do nosso atual sistema numérico decimal posicional com nove símbolos para os números $1,2,3, \ldots, 9$ e o zero, chamado pelos indianos de sunya.

Referências a conceitos geométricos e trigonométricos da matemática grega misturadas à tradição aritmética babilônica são encontrados em textos indianos consolidados. Sabe-se que as expedições persas de Ciro, em cerca de 500 a.C., e gregas de Alexandre, entre 327 e 326 a.C., alcançaram o vale do Indo (noroeste indiano), favorecendo a formação do que poderíamos chamar de uma comunidade científica internacional e o intercâmbio de conhecimento entre essas civilizações. Resultados dessa interação podem ser observados na divisão do círculo em 360 unidades (graus) e no uso de funções circulares semelhantes às utilizadas na Grécia em cálculos trigonométricos. Grande parte dessas ideias foram herdadas de trabalhos anteriores a Ptolomeu (c. 100 d.C.), com destaque especial para Hiparco de Rhodes (c. 150 a.C.), considerado o maior astrônomo da antiguidade e o precursor da trigonometria. Apesar de tudo, muito nesse campo ainda é especulação e não está totalmente claro para os historiadores da ciência até que ponto a influência dessas civilizações foi decisiva para o desenvolvimento da matemática indiana.

Hiparco, bem como os astrônomos babilônicos antes dele, supunha um modelo geocêntrico no qual os demais corpos celestes giravam ao redor da Terra em órbitas circulares, com movimento uniforme. Entretanto, ao analisar mais cuidadosamente o movimento do Sol, Hiparco fez novas descobertas. Ele observou que o tempo gasto pelo Sol do equinócio de primavera até o de outono é aproximadamente 186 dias, restando apenas 179 dias para a outra parte da órbita solar ao longo da eclíptica, assim chamada a órbita do Sol em torno da Terra. Isso significaria que, em média, o Sol desloca-se mais lentamente entre março e setembro do que no restante do ano! Como explicar essa observação? Hiparco não estava pronto para romper com a tradição dos movimentos circulares uniformes, e a única forma que encontrou para conciliar tais hipóteses com as observações foi propor que a Terra não ocuparia o centro dessa órbita. Movendo a Terra para uma posição excêntrica, afastando-a do Sol na primavera, Hiparco pôde efetivamente prolongar essa estação.

Mas qual a posição exata da Terra? Para responder essa pergunta Hiparco inventou a primeira função trigonométrica da história: a corda subtendida pelo ângulo, fundando a disciplina que hoje conhecemos como trigonometria; para saber como Hiparco resolveu o problema citado, o leitor interessado pode consultar [7]. Da sua parte, os indianos julgaram mais conveniente o uso da meia-corda para o estudo dos movimentos planetários, a qual chamavam de ardhajya ou jyardha ou bhujaya, e também era abreviada para $j y a^{2}$. Comparada a nossa atual função seno ${ }^{3}$, vale a relação (veja a Figura 1)

$$
\text { jya }(\alpha)=\frac{1}{2} \operatorname{crd}(2 \alpha)=\mathrm{R} \operatorname{sen}(\alpha) .
$$

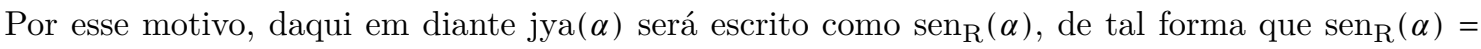
$\mathrm{R} \operatorname{sen}(\alpha)$, em que sen é o seno atual.

\footnotetext{
${ }^{1}$ Versão romanizada deste e dos próximos nomes dos indianos pelos padrões ISO 15919 e IAST. Para mais informações sobre o tema o leitor interessado pode consultar [6]

${ }^{2}$ Uma vez que os árabes receberam os trabalhos indianos, eles escreviam este termo como $j b$. Posteriormente eles passaram a chamar jya como jaib, que significa peitoral, seio. Os europeus, ao terem acesso aos trabalhos árabes, transcreveram jaib para sinus, que significa litoral, daí a etimologia do termo seno usado hoje em dia.

${ }^{3} \mathrm{~A}$ atual definição do seno como o cateto oposto sobre a hipotenusa em um triângulo retângulo é devida ao
} 


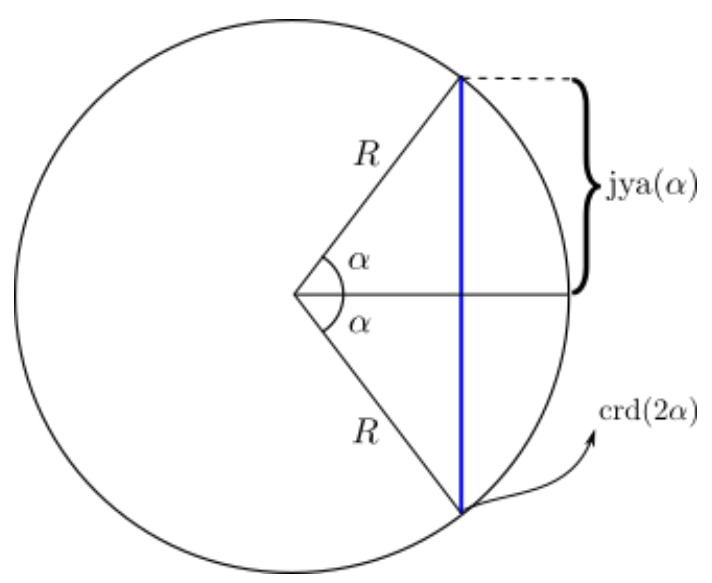

Figura 1: As funções circulares grega e indiana: corda e jya.

Vale notar que o seno indiano depende tanto do ângulo como do raio do círculo em questão. Em geral, o raio era escolhido de maneira apropriada para facilitar os cálculos. Em trabalhos indianos era muito comum o uso do raio do círculo com valor $\mathrm{R}=3438$. A explicação para esse número encontra-se na observação que a circunferência tem $360 \cdot 60=21.600$ minutos. Usando suas estimativas mais acuradas de $\pi$ (em [1], seção II, estrofe 10, temos $\pi=3$, 1416) igualando $21.600=2 \pi \mathrm{R}$, encontravam o valor acima para R. O uso das mesmas unidades para medir o arco e o raio do círculo ${ }^{4}$ garante que uma importante propriedade da função seno vale nesse contexto: $\operatorname{sen}(\theta) \approx \theta$, se $\theta$ é pequeno. Conforme veremos nesse trabalho, essa primeira estimativa é de vital importância para o cálculo das séries de potência do seno e do cosseno.

\section{Ariabata I e o método das diferenças}

Pouco se sabe sobre Ariabata I. É dele o livro Aryabhatiya, o mais antigo sobre matemática e astronomia indiana de um autor identificável [4]. Esse livro tem 4 seções e 123 estrofes, escrito dessa forma para ser simples e para facilitar a memorização. Nas estrofes 11 e 12 da segunda seção, ele escreve [1]:

11. Divida um quadrante de um círculo [em quantas partes desejar]. Então, dos triângulos [retângulos] e dos quadriláteros, pode-se encontrar tantos $\operatorname{sen}_{\mathrm{R}}(\theta)$ de $\operatorname{arcos}$ iguais quanto se deseja...

12. O primeiro $\operatorname{sen}_{R}(\theta)$ dividido por ele mesmo e diminuído pelo quociente resulta na segunda diferença de senos. $O$ primeiro $\operatorname{sen}_{R}(\theta)$, diminuído pelos quocientes obtidos dividindo cada um dos $\operatorname{sen}_{R}(\theta)$ anteriores pelo primeiro $\operatorname{sen}_{R}(\theta)$, resulta nas diferenças de senos restantes.

\footnotetext{
matemático suíço Loenhard Euler (1707-1783).

${ }^{4}$ Desde os primeiros astrônomos da antiguidade até Isaac Newton no século XVII, o grau representava uma medida de comprimento, não de ângulos. Para eles, por exemplo, $90^{\circ}$ representava o comprimento do quarto de círculo, não a medida de um ângulo reto.
} 
Na estrofe 11 ele descreve como eram construídos os elementos necessários para o cálculo de sua tabela de senos, considerando a divisão do quadrante em 24 partes iguais. Aqui encontramos mais uma vez a influência de Hiparco, que fazia divisões do quadrante da mesma maneira, considerando, porém, apenas 12 divisões de 450 minutos. É provável que Hiparco tenha sugerido essa divisão porque podemos realizá-la com o mínimo de esforço utilizando apenas a régua e o compasso euclidianos: começamos construindo um triângulo equilátero (primeira proposição dos Elementos de Euclides) para obter o ângulo de $60^{\circ}$; dividindo esse ângulo ao meio, o primeiro quadrante fica dividido em 3 partes iguais $\left(3 \times 30^{\circ}=90^{\circ}\right)$ e, daí, construindo bissetrizes sucessivamente, é possível dividi-lo em 6 partes iguais, depois 12, depois 24 .

$\mathrm{Na}$ estrofe 12 ele descreve o método de como obter os senos correspondentes a essas divisões. O primeiro $\operatorname{sen}_{\mathrm{R}}$ ele assume como o próprio valor do arco em minutos, 225. Para obter o segundo $\operatorname{sen}_{R}$, subtraia 225 de 225, resultando em 0; divida 225 por 225 e o quociente é 1 . A soma de 0 e 1 é subtraída de 225 e o resultado é 224 . Com isso calculamos a diferença entre o segundo $\operatorname{sen}_{R}$ e o primeiro (primeira diferença de senos), logo, o segundo seno é $225+224=449$. Continuando, subtraia 224 de 225, resultando em 1. Divida 449 por 225 e o quociente inteiro mais próximo é 2; subtraia $2+1$ de 225 e a diferença do terceiro para o segundo sen ${ }_{\mathrm{R}}$ é 222 , logo, o terceiro seno é $449+222=671$, e assim por diante.

Ariabata registra os 24 valores de $\operatorname{sen}_{\mathrm{R}}$ em minutos no livro. Há algumas disparidades caso se siga o método à risca, pois provavelmente ele efetuou algumas correções nos casos dos quocientes aproximados, embora nada no livro indique isso [1, p.29].

\begin{tabular}{|c|c|c|c|c|c|c|}
\hline $\mathbf{i}$ & $\mathbf{i} \Delta \boldsymbol{\theta}$ & $\Delta_{\mathbf{i}}$ & $\operatorname{sen}_{\mathbf{R}}(\mathbf{i})$ & $\frac{\operatorname{sen}_{\mathbf{R}}(\mathbf{i})}{\mathbf{R}}$ & $\operatorname{sen}(\mathbf{i})$ & $\varepsilon_{\mathbf{i}}=\frac{\operatorname{sen}_{\mathbf{R}}(\mathbf{i})}{\mathbf{R}}-\mathbf{s e n}(\mathbf{i})$ \\
\hline 1 & 225 & 225 & 225 & 0.065445026 & 0.065403129 & 0.00004190 \\
\hline 2 & 450 & 224 & 449 & 0.130599186 & 0.130526192 & 0.00007299 \\
\hline 3 & 675 & 222 & 671 & 0.195171611 & 0.195090322 & 0.00008129 \\
\hline 4 & 900 & 219 & 890 & 0.258871437 & 0.258819045 & 0.00005239 \\
\hline \multicolumn{7}{|c|}{$\vdots$} \\
\hline 21 & 4725 & 51 & 3372 & 0.980802792 & 0.98078528 & 0.00001751 \\
\hline 22 & 4950 & 37 & 3409 & 0.99156486 & 0.991444861 & 0.00012000 \\
\hline 23 & 5175 & 22 & 3431 & 0.997963933 & 0.997858923 & 0.00010501 \\
\hline 24 & 5400 & 7 & 3438 & 1 & 1 & 0 \\
\hline
\end{tabular}

Tabela 1: Tabela de senos de Ariabata.

A partir de Aryabhatiya, as próximas gerações de matemáticos realizaram diversos comentários para dar embasamento e também aprimorar a técnica. Entre eles destacam-se Bhaskara I [Bhāskhara] (c.500 d.C.), Brahmagupta e Nilakanta [Nīlakạ̣ṭha] (1444). Será a partir do método desse último que faremos um estudo do método das diferenças.

\subsection{Construção de tabela trigonométrica pelo método das diferenças}

No diagrama 2, os pontos $A_{i}, i=1,2,3, \ldots 24$ definem ângulos iguais a $\Delta \theta=225^{\prime}$. Seja $\operatorname{sen}_{R}(i)=$ $\operatorname{sen}_{\mathrm{R}}(\mathrm{i} \Delta \theta)$ e a diferença $\Delta_{\mathrm{i}}=\Delta \operatorname{sen}_{\mathrm{R}}(\mathrm{i})=\operatorname{sen}_{\mathrm{R}}(\mathrm{i})-\operatorname{sen}_{\mathrm{R}}(\mathrm{i}-1)$. Com isso, temos que

$$
\Delta_{i+1}=\Delta \operatorname{sen}_{R}(i+1)=\operatorname{sen}_{R}(i+1)-\operatorname{sen}_{R}(i)=P_{i+1} A_{i+1}-P_{i} A_{i}=C A_{i+1} .
$$


Sejam os pontos $B_{i}$ o ponto médio do arco $A_{i} A_{i-1}$, ou seja, são os pontos que definem a próxima quantidade de divisões. Com isso observe que $\triangle \mathrm{OB}_{\mathrm{i}+1} \mathrm{~T}_{\mathrm{i}+1}$ e $\triangle \mathrm{A}_{\mathrm{i}} \mathrm{CA}_{\mathrm{i+1}}$ são semelhantes, pois os três pares de lados homólogos são perpendiculares. Pela relação de semelhança e observando que $\mathrm{A}_{\mathrm{i}} \mathrm{A}_{\mathrm{i}+1}=\mathrm{B}_{\mathrm{i}} \mathrm{B}_{\mathrm{i}+1}=\operatorname{crd}(\Delta \theta)=\operatorname{crd}(1)$, em que $\operatorname{crd}(\alpha)$ é a corda definida pelo arco $\alpha$. Temos que

$$
\frac{\mathrm{CA}_{\mathrm{i}+1}}{\mathrm{OT}_{\mathrm{i}+1}}=\frac{\mathrm{A}_{1} \mathrm{~A}_{\mathrm{i}+1}}{\mathrm{OB}_{\mathrm{i}+1}}=\frac{\mathrm{CA}_{\mathrm{i}}}{\mathrm{T}_{\mathrm{i}+1} \mathrm{~B}_{\mathrm{i}+1}},
$$

de onde obtemos, pela primeira igualdade,

$$
\frac{\Delta_{\mathrm{i}+1}}{\mathrm{OT}_{\mathrm{i}+1}}=\frac{\operatorname{crd}(1)}{\mathrm{R}},
$$

ou seja,

$$
\Delta_{\mathrm{i}+1}=\frac{\operatorname{crd}(1)}{\mathrm{R}} \mathrm{OT}_{\mathrm{i}+1} .
$$

De maneira análoga obtemos

$$
\Delta_{\mathrm{i}}=\frac{\operatorname{crd}(1)}{\mathrm{R}} \mathrm{OT}_{\mathrm{i}},
$$

e, daí,

$$
\Delta_{\mathrm{i}}-\Delta_{\mathrm{i}+1}=\frac{\operatorname{crd}(1)}{\mathrm{R}}\left(\mathrm{OT}_{\mathrm{i}}-\mathrm{OT}_{\mathrm{i}+1}\right)=\frac{\operatorname{crd}(1)}{\mathrm{R}} \mathrm{T}_{\mathrm{i}+1} \mathrm{~T}_{\mathrm{i}} .
$$

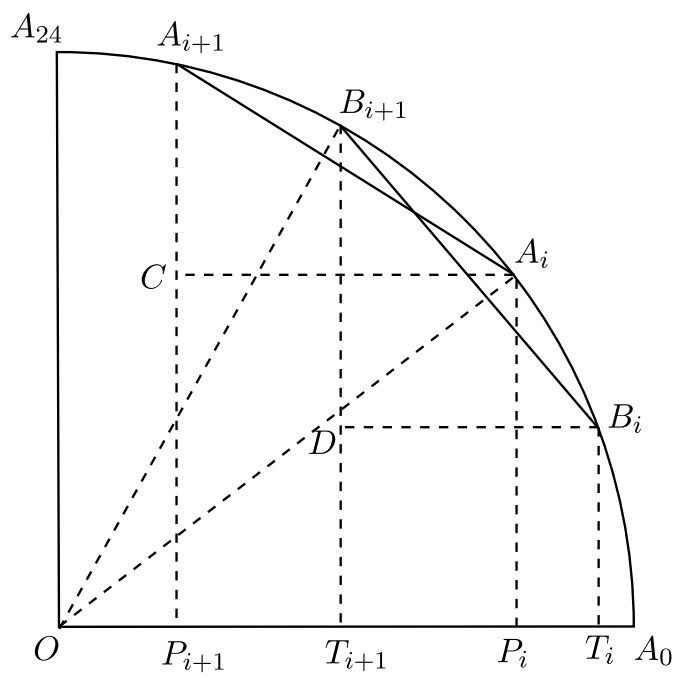

Figura 2: Método das diferenças.

Temos também em (2) que os triângulos $\triangle \mathrm{B}_{\mathrm{i}} \mathrm{DB}_{\mathrm{i}+1}$ e $\triangle \mathrm{A}_{\mathrm{i}} \mathrm{P}_{\mathrm{i}} \mathrm{O}$ são semelhantes pelo mesmo motivo explicado anteriormente. Portanto,

$$
\frac{\mathrm{B}_{\mathrm{i}} \mathrm{D}}{\mathrm{B}_{\mathrm{i}} \mathrm{B}_{\mathrm{i}+1}}=\frac{\mathrm{A}_{\mathrm{i}} \mathrm{P}_{\mathrm{i}}}{\mathrm{A}_{\mathrm{i}} \mathrm{O}}
$$

ou seja,

$$
\mathrm{T}_{\mathrm{i}+1} \mathrm{~T}_{\mathrm{i}}=\mathrm{B}_{\mathrm{i}} \mathrm{D}=\frac{\operatorname{crd}(1)}{\mathrm{R}} \operatorname{sen}_{\mathrm{R}}(\mathrm{i}) .
$$


Substituindo em (2), vem que

$$
\frac{\Delta_{\mathrm{i}}-\Delta_{\mathrm{i}+1}}{\operatorname{sen}_{\mathrm{R}}(\mathrm{i})}=\left[\frac{\operatorname{crd}(1)}{\mathrm{R}}\right]^{2}
$$

Esse resultado mostra que a razão da segunda diferença por seu sen $\mathrm{R}_{\mathrm{R}}$ é constante, o que nos leva a uma fórmula de recorrência. Até que ponto Ariabata estava ciente dessa propriedade, não se sabe. Igualando a expressão do lado esquerdo com ela própria no caso i $=1$, encontra-se a seguinte fórmula fundamental

$$
\frac{\Delta_{\mathrm{i}}-\Delta_{\mathrm{i}+1}}{\operatorname{sen}_{\mathrm{R}}(\mathrm{i})}=\frac{\Delta_{1}-\Delta_{2}}{\operatorname{sen}_{\mathrm{R}}(1)}
$$

ou, ainda,

$$
\Delta_{\mathrm{i}}-\Delta_{\mathrm{i}+1}=\left(\Delta_{1}-\Delta_{2}\right) \frac{\operatorname{sen}_{\mathrm{R}}(\mathrm{i})}{\operatorname{sen}_{\mathrm{R}}(1)} .
$$

Dados os valores de $\operatorname{sen}_{R}(1)$ e $\Delta=\Delta_{1}-\Delta_{2}$ a equação (3) permite calcular todos os 23 senos restantes. De fato, lembrando que $\operatorname{sen}_{\mathrm{R}}(0)=0$, somando as equações

$$
\begin{aligned}
\Delta_{1}= & \operatorname{sen}_{\mathrm{R}}(1)-\operatorname{sen}_{\mathrm{R}}(0), \\
\Delta_{2}= & \operatorname{sen}_{\mathrm{R}}(2)-\operatorname{sen}_{\mathrm{R}}(1), \\
\Delta_{3}= & \operatorname{sen}_{\mathrm{R}}(3)-\operatorname{sen}_{\mathrm{R}}(2), \\
& \vdots \\
\Delta_{\mathrm{n}-1}= & \operatorname{sen}_{\mathrm{R}}(\mathrm{n}-1)-\operatorname{sen}_{\mathrm{R}}(\mathrm{n}-2), \\
\Delta_{\mathrm{n}}= & \operatorname{sen}_{\mathrm{R}}(\mathrm{n})-\operatorname{sen}_{\mathrm{R}}(\mathrm{n}-1)
\end{aligned}
$$

e cancelando termos semelhantes concluímos que

$$
\operatorname{sen}_{\mathrm{R}}(\mathrm{n})=\Delta_{1}+\Delta_{2}+\Delta_{3}+\cdots+\Delta_{\mathrm{n}}
$$

Para calcular as diferenças observamos inicialmente que

$$
\begin{aligned}
& \Delta_{1}=\operatorname{sen}_{\mathrm{R}}(1)-\operatorname{sen}_{\mathrm{R}}(0)=\operatorname{sen}_{\mathrm{R}}(1), \\
& \Delta_{2}=\Delta_{1}-\Delta=\operatorname{sen}_{\mathrm{R}}(1)-\Delta,
\end{aligned}
$$

com as quais já podemos calcular $\operatorname{sen}_{\mathrm{R}}(2)$ da maneira que foi descrita por Ariabata. Agora, usando (3), calculamos $\Delta_{3}$ como

$$
\Delta_{3}=\Delta_{2}-\Delta \frac{\operatorname{sen}_{R}(2)}{\operatorname{sen}_{R}(1)}=\operatorname{sen}_{R}(1)-\Delta\left(\frac{\operatorname{sen}_{R}(1)+\operatorname{sen}_{R}(2)}{\operatorname{sen}_{R}(1)}\right),
$$

e com $\Delta_{1}, \Delta_{2}$ e $\Delta_{3}$ calculamos $\operatorname{sen}_{\mathrm{R}}(3)$. Para $\Delta_{4}$ temos

$$
\Delta_{4}=\Delta_{3}-\Delta \frac{\operatorname{sen}_{\mathrm{R}}(3)}{\operatorname{sen}_{\mathrm{R}}(1)}=\operatorname{sen}_{\mathrm{R}}(1)-\Delta\left(\frac{\operatorname{sen}_{\mathrm{R}}(1)+\operatorname{sen}_{\mathrm{R}}(2)+\operatorname{sen}_{\mathrm{R}}(3)}{\operatorname{sen}_{\mathrm{R}}(1)}\right) .
$$

Com $\Delta_{1}, \Delta_{2}, \Delta_{3}$ e $\Delta_{4}$ podemos calcular $\operatorname{sen}_{\mathrm{R}}(4)$ e assim prosseguimos. Confirmando o resultado usando indução, assuma que a recorrência vale para n. Como

$$
\Delta_{\mathrm{n}}=\operatorname{sen}_{\mathrm{R}}(1)-\Delta\left(\frac{\sum_{\mathrm{k}=1}^{\mathrm{n}-1} \operatorname{sen}_{\mathrm{R}}(\mathrm{k})}{\operatorname{sen}_{\mathrm{R}}(1)}\right)
$$


a partir de (3) temos que

$$
\begin{aligned}
& \Delta_{\mathrm{n}}-\Delta_{\mathrm{n}+1}=\Delta \frac{\operatorname{sen}_{\mathrm{R}}(\mathrm{n})}{\operatorname{sen}_{\mathrm{R}}(1)} \\
& \Delta_{\mathrm{n}+1}=\Delta_{\mathrm{n}}-\Delta \frac{\operatorname{sen}_{\mathrm{R}}(\mathrm{n})}{\operatorname{sen}_{\mathrm{R}}(1)} \\
& \Delta_{\mathrm{n}+1}=\operatorname{sen}_{\mathrm{R}}(1)-\Delta\left(\frac{\sum_{\mathrm{k}=1}^{\mathrm{n}-1} \operatorname{sen}_{\mathrm{R}}(\mathrm{k})}{\operatorname{sen}_{\mathrm{R}}(1)}\right)-\Delta \frac{\operatorname{sen}_{\mathrm{R}}(\mathrm{n})}{\operatorname{sen}_{\mathrm{R}}(1)} \\
& \Delta_{\mathrm{n}+1}=\operatorname{sen}_{\mathrm{R}}(1)-\Delta\left(\frac{\sum_{\mathrm{k}=1}^{\mathrm{n}} \operatorname{sen}_{\mathrm{R}}(\mathrm{k})}{\operatorname{sen}_{\mathrm{R}}(1)}\right) .
\end{aligned}
$$

Na tabela (1) encontramos um excerto da tabela de senos de Ariabata. As colunas à direita comparam os valores do seno moderno pelos cálculos de Ariabata com aqueles que obtemos com a calculadora moderna.

O método das diferenças virou o padrão indiano para construção de tabelas de sen ${ }_{\mathrm{R}}$, e os matemáticos que vieram após trouxeram várias melhorias e refinamentos. Regra geral, os aprimoramentos consistiam na utilização de métodos de interpolação, isto é, obtenção de novos valores de $\operatorname{sen}_{R}$ a partir dos valores já conhecidos. Brahmagupta, por exemplo, enunciou um resultado que em notação moderna pode ser enunciado como:

$$
\operatorname{sen}_{\mathrm{R}}(\mathrm{i} \Delta \theta+\alpha)=\operatorname{sen}_{\mathrm{R}}(\mathrm{i})+\frac{\alpha}{2 \Delta \theta}\left(\Delta_{\mathrm{i}}+\Delta_{\mathrm{i}+1}\right)-\frac{\alpha^{2}}{2 \Delta \theta^{2}}\left(\Delta_{\mathrm{i}}-\Delta_{\mathrm{i}+1}\right),
$$

com $\alpha<\Delta \theta$. Tal aproximação é um caso particular da fórmula conhecida como interpolação de Newton-Stirling de segunda ordem [2].

Bhaskara I (c.700 d.C), da sua parte, encontrou uma fração racional (quociente de dois polinômios) cujos valores são notavelmente próximos de $\operatorname{sen}_{\mathrm{R}}$. Para $\mathrm{R}=1$, essa função é dada como

$$
\mathrm{p}(\mathrm{x})=\frac{4 \mathrm{x}(180-\mathrm{x})}{[40500-\mathrm{x}(180-\mathrm{x})]},
$$

em que $\mathrm{x}$ é medido em graus e $0 \leq \mathrm{x} \leq 180$. Nesse intervalo, a fórmula leva a um erro de no máximo 1\%. Podemos ter uma ideia da precisão da fórmula de Bhaskara I observando a Figura 3 onde foram plotadas as funções s e sen. Para mais detalhes sobre o método utilizado por Bhaskara para chegar a sua fórmula veja [7].

Por fim, Vatesvara [Vațeśvara] (880 d.C.), utilizando métodos de aproximação, reconstruiu a tabela de Ariabata com 96 valores de $\operatorname{sen}_{\mathrm{R}}$ utilizando $\mathrm{R}=3437^{\prime} 44^{\prime \prime}$. Esse refinamento no valor de $\mathrm{R}$ implica uma aproximação de $\pi$ ainda mais precisa que seus antecessores.

Por meio dos trabalhos inovadores de Ariabata e seus conterrâneos, foi possível estabelecer séries de potência para funções trigonométricas conforme discutiremos a seguir. Vale citar que os indianos já trabalhavam com cálculos envolvendo séries convergentes, principalmente no período em que Madhava [Mādhava] (1340 d.C.) iniciou o que se conhece hoje como Escola de Kerala: gerações de cientistas brilhantes que tiveram grande produção de trabalhos astronômicos e matemáticos, antecipando europeus em vários resultados; particularmente as séries numéricas de $\pi$, e as séries de potências para o seno e o cosseno. Uma descrição mais detalhada dos resultados da Escola de Kerala é feita em [7]. 


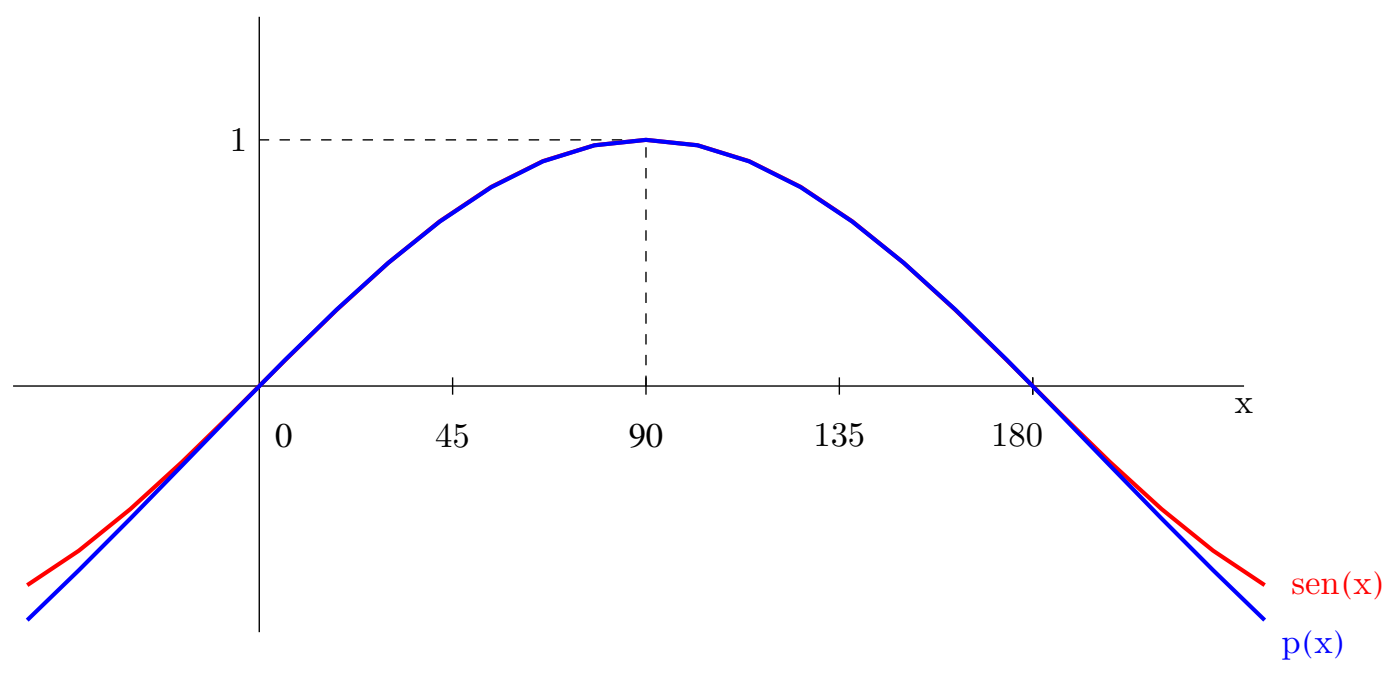

Figura 3: As funções sen(x) e p(x).

\section{Séries de potências de seno e cosseno}

Seguiremos as ideias descritas no livro Yuktibhasa de Jyestadeva [Jyeșṭhadeva] (1500 d.C.), ma-

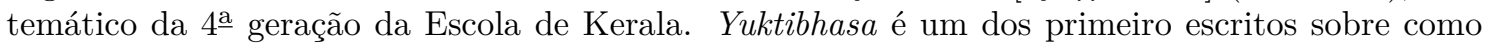
obter tais séries, embora todo o mérito seja atribuído a Madhava. Nesse ponto tomaremos $\mathrm{R}=1$, caso em que $\operatorname{sen}_{\mathrm{R}}(\alpha)=\operatorname{sen}(\alpha)$, e assumiremos que todas as funções trigonométricas envolvidas utilizam medidas angulares em radianos a fim de evitar uma série de constantes adicionais que surgem naturalmente quando utilizamos a função seno indiana.

Em relação à figura 2 , note que se definimos $\angle \mathrm{A}_{0} \mathrm{OA}_{\mathrm{i}}=\mathrm{i} \Delta \theta=\theta$ e pomos $\operatorname{sen}(1)=\operatorname{sen}(\Delta \theta) \approx \Delta \theta$, a equação (1) pode ser reescrita como

$$
\frac{\Delta \operatorname{sen}(\theta)}{\cos (\theta)}=\frac{\Delta \theta}{1}=\frac{\Delta \cos (\theta)}{\operatorname{sen}(\theta)}
$$

em que $\Delta \cos (\theta)=\cos (\theta)-\cos (\theta+\Delta \theta)$. Nesse momento é preciso observar que foi feita a aproximação $\cos \left(\theta+\frac{\Delta \theta}{2}\right) \approx \cos (\theta)$, assim $\operatorname{como} \operatorname{sen}\left(\theta-\frac{\Delta \theta}{2}\right) \approx \operatorname{sen}(\theta)$. Em seu trabalho, Jyestadeva não deixa claro essa aproximação, porém sua validade não é duvidada à medida que se fazem mais divisões no quadrante, fato que implica $\Delta \theta \rightarrow 0$. Da equação anterior obtemos

$$
\begin{aligned}
& \Delta \operatorname{sen}(\theta)=\cos (\theta) \Delta \theta \\
& \Delta \cos (\theta)=\operatorname{sen}(\theta) \Delta \theta .
\end{aligned}
$$

Do ponto de vista moderno, as relações acima são aplicações do Teorema do Valor Médio para as funções seno e cosseno. A diferença de sinal que observamos quando comparadas às fórmulas atuais deve-se ao fato de ser a definição de $\Delta$ cos invertida em relação com $\Delta$ sen, a fim de evitar valores negativos causados pelo decrescimento do cosseno no primeiro quadrante.

Nos trabalhos indianos as séries de potência são obtidas simultaneamente. A aproximação de primeira ordem do $\operatorname{seno}(\operatorname{sen}(\theta) \approx \theta)$ é usada para obter a aproximação de segunda ordem do cosseno, que será usada para obter a aproximação de terceira ordem do seno, e assim por diante. 
Para isso, considere a Figura 4. Nela temos que $\theta=\mathrm{n} \Delta \theta, \operatorname{sen}(\mathrm{i})=\mathrm{A}_{\mathrm{i}} \mathrm{P}_{\mathrm{i}}$ e $\cos (\mathrm{i})=\mathrm{OP}_{\mathrm{i}}$. Com isso,

$$
1-\cos (\theta)=\mathrm{A}_{0} \mathrm{P}_{\mathrm{n}}=\mathrm{P}_{0} \mathrm{P}_{1}+\mathrm{P}_{1} \mathrm{P}_{2}+\mathrm{P}_{2} \mathrm{P}_{3}+\mathrm{P}_{3} \mathrm{P}_{4}+\cdots+\mathrm{P}_{\mathrm{n}-1} \mathrm{P}_{\mathrm{n}},
$$

$\operatorname{mas} \mathrm{P}_{\mathrm{i}-1} \mathrm{P}_{\mathrm{i}}=\Delta \cos (\mathrm{i})=\operatorname{sen}(\mathrm{i}) \Delta \theta, \log \mathrm{O}$,

$$
\cos (\theta)=1-[\operatorname{sen}(1)+\operatorname{sen}(2)+\cdots+\operatorname{sen}(n)] \Delta \theta .
$$

Usando a aproximação de primeira $\operatorname{ordem} \operatorname{sen}(\theta) \approx \theta$ e a igualdade $\Delta \theta=\frac{\theta}{\mathrm{n}}$ vem que

$$
\begin{aligned}
\cos \theta & =1-[\Delta \theta+2 \Delta \theta+\cdots+\mathrm{n} \Delta \theta] \Delta \theta \\
& =1-[1+2+\cdots+\mathrm{n}] \Delta \theta^{2} \\
& \approx 1-\frac{\mathrm{n}^{2}}{2} \Delta \theta^{2} \\
& =1-\frac{\theta^{2}}{2 !}
\end{aligned}
$$

Aqui foi utilizada a aproximação

$$
1+2+\cdots+\mathrm{n} \approx \frac{\mathrm{n}^{2}}{2}
$$

Que é válida para $\mathrm{n}$ - número de divisões do quadrante - grande. Essas somas eram conhecidas anteriormente pelos árabes. O matemático egípcio Abu al-Haytham [Abū 'Al̄̄ al Hasan] (965-1039) mostrou que

$$
1^{\mathrm{p}}+2^{\mathrm{p}}+3^{\mathrm{p}}+\cdots+\mathrm{n}^{\mathrm{p}}=\frac{\mathrm{n}^{\mathrm{p}+1}}{\mathrm{p}+1}+\frac{\mathrm{n}^{\mathrm{p}}}{2}+\mathrm{r}(\mathrm{n}),
$$

em que r(n) é um polinômio em n de grau menor do que p. Da expressão acima é possível concluir que para n suficientemente grande temos, em geral,

$$
1^{\mathrm{p}}+2^{\mathrm{p}}+3^{\mathrm{p}}+\cdots+\mathrm{n}^{\mathrm{p}} \approx \frac{\mathrm{n}^{\mathrm{p}+1}}{\mathrm{p}+1} .
$$

A fim de tornar a presente discussão mais completa, interrompemos brevemente a dedução das séries de potência para explicar o método indiano para verificar (6). Inicialmente, defina

$$
\mathrm{S}_{\mathrm{n}}^{(\mathrm{p})}=1^{\mathrm{p}}+2^{\mathrm{p}}+3^{\mathrm{p}}+\cdots+\mathrm{n}^{\mathrm{p}}
$$

Daí temos que

$$
\begin{aligned}
\mathrm{nS}_{\mathrm{n}}^{(\mathrm{p}-1)} & =\mathrm{n}\left(1^{\mathrm{p}-1}+2^{\mathrm{p}-1}+\cdots+\mathrm{n}^{\mathrm{p}-1}\right) \\
& =\mathrm{n}^{\mathrm{p}}+\mathrm{n}\left[1^{\mathrm{p}-1}+2^{\mathrm{p}-1}+\cdots+(\mathrm{n}-1)^{\mathrm{p}-1}\right] \\
& =\mathrm{n}^{\mathrm{p}}+\mathrm{nS}_{\mathrm{n}-1}^{(\mathrm{p}-1)} \\
& =\mathrm{n}^{\mathrm{p}}+[(\mathrm{n}-1)+1] \mathrm{S}_{\mathrm{n}-1}^{(\mathrm{p}-1)} \\
& =\mathrm{n}^{\mathrm{p}}+(\mathrm{n}-1) \mathrm{S}_{\mathrm{n}-1}^{(\mathrm{p}-1)}+\mathrm{S}_{\mathrm{n}-1}^{(\mathrm{p}-1)} \\
& =\mathrm{n}^{\mathrm{p}}+(\mathrm{n}-1)^{\mathrm{p}}+(\mathrm{n}-1) \mathrm{S}_{\mathrm{n}-2}^{(\mathrm{p}-1)}+\mathrm{S}_{\mathrm{n}-1}^{(\mathrm{p}-1)} \\
& =\mathrm{n}^{\mathrm{p}}+(\mathrm{n}-1)^{\mathrm{p}}+(\mathrm{n}-2)^{\mathrm{p}}+(\mathrm{n}-3) \mathrm{S}_{\mathrm{n}-3}^{(\mathrm{p}-1)}+\mathrm{S}_{\mathrm{n}-2}^{(\mathrm{p}-1)}+\mathrm{S}_{\mathrm{n}-1}^{(\mathrm{p}-1)}
\end{aligned}
$$

Prosseguindo dessa forma chegamos a

$$
\mathrm{nS}_{\mathrm{n}}^{(\mathrm{p}-1)}=\mathrm{S}_{\mathrm{n}}^{(\mathrm{p})}+\mathrm{S}_{\mathrm{n}-1}^{(\mathrm{p}-1)}+\mathrm{S}_{\mathrm{n}-2}^{(\mathrm{p}-1)}+\cdots+\mathrm{S}_{2}^{(\mathrm{p}-1)}+\mathrm{S}_{1}^{(\mathrm{p}-1)} .
$$


Para $\mathrm{p}=1$, essa última equação dá

$$
\mathrm{nS}_{\mathrm{n}}^{(0)}=\mathrm{S}_{\mathrm{n}}^{(1)}+\mathrm{S}_{\mathrm{n}-1}^{(0)}+\mathrm{S}_{\mathrm{n}-2}^{(0)}+\cdots+\mathrm{S}_{2}^{(0)}+\mathrm{S}_{1}^{(0)}
$$

ou seja:

$$
\begin{aligned}
\mathrm{n}^{2} & =\mathrm{S}_{\mathrm{n}}^{(1)}+(\mathrm{n}-1)+(\mathrm{n}-2)+\cdots+2+1 \\
& =\mathrm{S}_{\mathrm{n}}^{(1)}+\mathrm{S}_{\mathrm{n}-1}^{(1)} .
\end{aligned}
$$

Supondo que para $\mathrm{n}$ muito grande vale $\mathrm{S}_{\mathrm{n}}^{(1)} \approx \mathrm{S}_{\mathrm{n}-1}^{(1)}$, os cientistas indianos concluíram da expressão acima que $\mathrm{n}^{2} \approx 2 \mathrm{~S}_{\mathrm{n}}^{(1)}$, isto é,

$$
\mathrm{S}_{\mathrm{n}}^{(1)}=1+2+\cdots+\mathrm{n} \approx \frac{\mathrm{n}^{2}}{2}
$$

$\mathrm{O}$ caso $\mathrm{p}=2$ era obtido dessa primeira aproximação, mostrando-se inicialmente que para $\mathrm{n}$ grande

$$
\mathrm{S}_{\mathrm{n}-1}^{(1)}+\mathrm{S}_{\mathrm{n}-2}^{(1)}+\cdots+\mathrm{S}_{2}^{(1)}+\mathrm{S}_{1}^{(1)} \approx \frac{\mathrm{S}_{\mathrm{n}}^{(2)}}{2},
$$

de onde, usando (7), concluíam que

$$
\mathrm{n} \frac{\mathrm{n}^{2}}{2} \approx \mathrm{S}_{\mathrm{n}}^{(2)}+\frac{\mathrm{S}_{\mathrm{n}}^{(2)}}{2}=\frac{3}{2} \mathrm{~S}_{\mathrm{n}}^{(2)},
$$

isto é:

$$
\mathrm{S}_{\mathrm{n}}^{(2)}=1^{2}+2^{2}+\cdots+\mathrm{n}^{2} \approx \frac{\mathrm{n}^{3}}{3} .
$$

$\mathrm{O}$ caso $\mathrm{p}=3$ era obtido da mesma forma a partir do caso $\mathrm{p}=2$, e daí concluíam que, em geral, deveria valer

$$
\mathrm{S}_{\mathrm{n}}^{(\mathrm{p})}=1^{\mathrm{p}}+2^{\mathrm{p}}+\cdots+\mathrm{n}^{\mathrm{p}} \approx \frac{\mathrm{n}^{\mathrm{p}+1}}{\mathrm{p}+1} .
$$

Essas fórmulas foras enunciadas de maneira correta em meados do século XIV nos trabalhos de Narayana Pandita [Nārāyaṇa Pạ̣dita] (1325). O método explicado acima é encontrado em Yuktibhasa escrito por Jyestadeva por volta de 1500.

Finalizado esse interlúdio, seguimos a dedução das séries de potências de seno e cosseno. Seguindo para a aproximação de terceira ordem para o seno a partir da aproximação de segunda ordem para o cosseno, fazendo uma releitura das equações (4) exatamente como descrito na Figura 2, temos

$$
\begin{aligned}
\Delta \operatorname{sen}(1)= & \cos \left(1-\frac{1}{2}\right) \Delta \theta \\
\Delta \operatorname{sen}(2)= & \cos \left(2-\frac{1}{2}\right) \Delta \theta \\
\Delta \operatorname{sen}(3)= & \cos \left(3-\frac{1}{2}\right) \Delta \theta \\
& \vdots \\
\Delta \operatorname{sen}(n)= & \cos \left(n-\frac{1}{2}\right) \Delta \theta
\end{aligned}
$$

Dessa forma vem que

$$
\Delta \operatorname{sen}(1)-\Delta \operatorname{sen}(\mathrm{n})=\left[\cos \left(\frac{1}{2}\right)-\cos \left(\mathrm{n}-\frac{1}{2}\right)\right] \Delta \theta .
$$

Usando as aproximações $\cos \left(\frac{1}{2}\right) \approx 1 \mathrm{e} \cos \left(\mathrm{n}-\frac{1}{2}\right) \approx \cos (\mathrm{n})$ obtemos

$$
\Delta \operatorname{sen}(1)-\Delta \operatorname{sen}(\mathrm{n}) \approx[1-\cos (\mathrm{n})] \Delta \theta \text {. }
$$




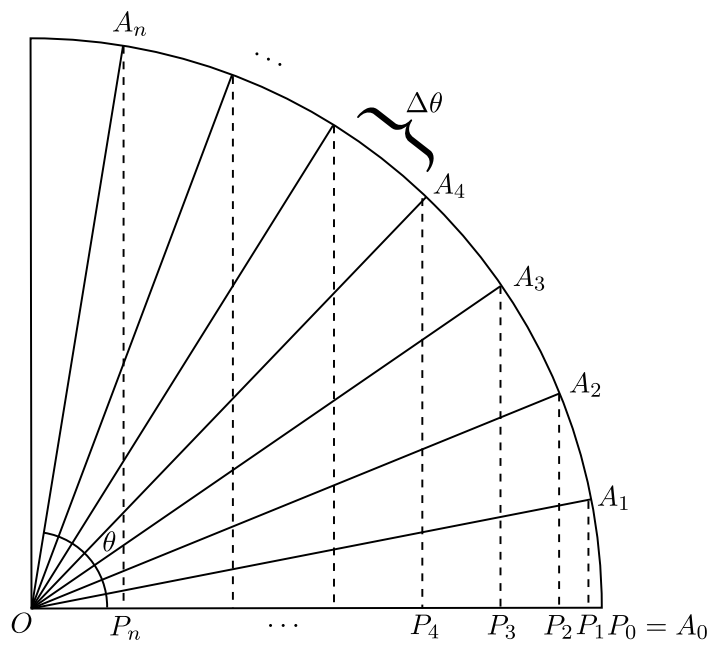

Figura 4: Divisão do quadrante em $\mathrm{n}=3 \cdot 2^{\mathrm{k}}$ partes.

Por fim, usando (5) obtemos

$$
[1-\cos (\mathrm{n})] \Delta \theta=[1-\cos (\mathrm{n} \Delta \theta)] \Delta \theta=\left[1-\left(1-\frac{\mathrm{n}^{2}}{2} \Delta \theta^{2}\right)\right] \Delta \theta=\frac{\mathrm{n}^{2}}{2} \Delta \theta^{3} .
$$

Fazendo o mesmo para as demais diferenças $\Delta \operatorname{sen}(1)-\Delta \operatorname{sen}(i)$ obtemos o seguinte:

$$
\begin{aligned}
\Delta \operatorname{sen}(1)-\Delta \operatorname{sen}(\mathrm{n}) & \approx \frac{\mathrm{n}^{2}}{2} \Delta \theta^{3}, \\
\Delta \operatorname{sen}(1)-\Delta \operatorname{sen}(\mathrm{n}-1) & \approx \frac{(\mathrm{n}-1)^{2}}{2} \Delta \theta^{3} . \\
\Delta \operatorname{sen}(1)-\Delta \operatorname{sen}(\mathrm{n}-2) & \approx \frac{(\mathrm{n}-2)^{2}}{2} \Delta \theta^{3} . \\
& \vdots \\
\Delta \operatorname{sen}(1)-\Delta \operatorname{sen}(2) & \approx \frac{2^{2}}{2} \Delta \theta^{3} .
\end{aligned}
$$

A soma procurada fica

$$
(n-1) \Delta \operatorname{sen}(1)-[\Delta \operatorname{sen}(2)+\Delta \operatorname{sen}(3)+\cdots+\Delta \operatorname{sen}(n)]=n \Delta \operatorname{sen}(1)-\sum_{i=1}^{n} \Delta \operatorname{sen}(i) .
$$

Lembrando que $\Delta \operatorname{sen}(1)=\operatorname{sen}(1)-\operatorname{sen}(0)=\operatorname{sen}(1) \approx \Delta \theta \mathrm{e}$

$$
\begin{aligned}
\sum_{\mathrm{i}-1}^{\mathrm{n}} \Delta \operatorname{sen}(\mathrm{i}) & =\Delta \operatorname{sen}(1)+\Delta \operatorname{sen}(2)+\Delta \operatorname{sen}(3)+\cdots+\Delta \operatorname{sen}(\mathrm{n}-1)+\Delta \operatorname{sen}(\mathrm{n}) \\
& =[\operatorname{sen}(1)-\operatorname{sen}(\theta)]^{0}+[\operatorname{sen}(2)-\operatorname{sen}(1)]+[\operatorname{sen}(3)-\operatorname{sen}(2)]+\cdots+ \\
& +[\operatorname{sen}(n-1)-\operatorname{sen}(n-2)]+[\operatorname{sen}(n)-\operatorname{sen}(n-1)] \\
= & \operatorname{sen}(n)=\operatorname{sen}(n \Delta \theta)=\operatorname{sen}(\theta),
\end{aligned}
$$


a soma dos lados esquerdos dá

$$
\mathrm{n} \Delta \theta-\operatorname{sen}(\theta)=\theta-\operatorname{sen}(\theta),
$$

Portanto, se somarmos os dois lados de (8) obtemos

$$
\theta-\operatorname{sen}(\theta)=\left[\mathrm{n}^{2}+(\mathrm{n}-1)^{2}+\cdots+2^{2}\right] \frac{\Delta \theta^{3}}{2} \approx \frac{\mathrm{n}^{3}}{3} \frac{\Delta \theta^{3}}{2}=\frac{(\mathrm{n} \Delta \theta)^{3}}{3 \cdot 2}=\frac{\theta^{3}}{3 !},
$$

de onde obtemos a aproximação de terceira ordem para o seno

$$
\operatorname{sen}(\theta)=\theta-\frac{\theta^{3}}{3 !}
$$

Para chegar à estimativa de segunda ordem do cosseno (5), aplicamos a estimativa de primeira $\operatorname{ordem} \operatorname{sen}(\theta) \approx \theta$. Jyestadeva acreditava que, ao se aplicar uma estimativa mais acurada, o valor resultante é também mais acurado, fato que o levou a uma recursão no processo de cálculo das suas séries de potência. Usando (9) vem

$$
\begin{aligned}
1-\cos (\theta) & =\left[\left(\Delta \theta-\frac{\Delta \theta^{3}}{3 !}\right)+\left(2 \Delta \theta-\frac{(2 \Delta \theta)^{3}}{3 !}\right)+\cdots+\left(\mathrm{n} \Delta \theta-\frac{(\mathrm{n} \Delta \theta)^{3}}{3 !}\right)\right] \\
& =[1+2+\cdots+\mathrm{n}] \Delta \theta^{2}-\left[1^{3}+2^{2}+\cdots+\mathrm{n}^{3}\right] \frac{\Delta \theta^{4}}{3 !} \\
& \approx \frac{\mathrm{n}^{2}}{2} \Delta \theta^{2}-\frac{\mathrm{n}^{4}}{4} \frac{\Delta \theta^{4}}{3 !}
\end{aligned}
$$

ou seja,

$$
\cos (\theta)=1-\frac{\theta^{2}}{2 !}+\frac{\theta^{4}}{4 !}
$$

Usando essa aproximação de quarta ordem para o cálculo das equações (8), podemos produzir uma aproximação de quinta ordem para o seno:

$$
\begin{aligned}
\theta-\operatorname{sen}(\theta) & =\left(\frac{1^{2}}{2} \Delta \theta^{3}-\frac{1^{4}}{4 !} \Delta \theta^{5}\right)+\left(\frac{2^{2}}{2} \Delta \theta^{3}-\frac{2^{4}}{4 !} \Delta \theta^{5}\right)+\cdots+\left(\frac{\mathrm{n}^{2}}{2} \Delta \theta^{3}-\frac{\mathrm{n}^{4}}{4 !} \Delta \theta^{5}\right) \\
& =\left(1^{2}+2^{2}+\cdots+\mathrm{n}^{2}\right) \frac{\Delta \theta^{3}}{2}-\left(1^{4}+2^{4}+\cdots+\mathrm{n}^{4}\right) \frac{\Delta \theta^{5}}{4 !} \\
& \approx \frac{\mathrm{n}^{3}}{3} \frac{\Delta \theta^{3}}{2}+\frac{\mathrm{n}^{5}}{5} \frac{\Delta \theta^{5}}{4 !},
\end{aligned}
$$

de onde concluímos que

$$
\operatorname{sen}(\theta)=\theta-\frac{\theta^{3}}{3 !}+\frac{\theta^{5}}{5 !}
$$

Se continuarmos nesse processo iterativo, produzimos estimativas de ordem superior para o seno e o cosseno acrescentando um termo a cada etapa. Com isso temos

$$
\begin{aligned}
& \operatorname{sen}(\theta)=\sum_{n=0}^{\infty} \frac{(-1)^{\mathrm{n}}}{(2 \mathrm{n}+1) !} \theta^{2 \mathrm{n}+1}=\theta-\frac{\theta^{3}}{3 !}+\frac{\theta^{5}}{5 !}-\frac{\theta^{7}}{7 !}+\cdots \\
& \cos (\theta)=\sum_{\mathrm{n}=0}^{\infty} \frac{(-1)^{\mathrm{n}}}{(2 \mathrm{n}) !} \theta^{2 \mathrm{n}}=1-\frac{\theta^{2}}{2 !}+\frac{\theta^{4}}{4 !}-\frac{\theta^{6}}{6 !}+\cdots
\end{aligned}
$$


As expressões acima, embora chamadas séries de Taylor em homenagem ao matemático britânico Brook Taylor (1685-1731), fizeram seu debute na Matemática europeia em uma carta escrita em 1676 pelo também britânico Isaac Newton (1642-1727) para o secretário da Royal Society de Londres Henry Oldenburg (1619-1677), mas destinada ao matemático alemão Gottfried Leibniz (1646-1716) em resposta a um pedido desse último por mais informações sobre o Cálculo.

Vale salientar que o matemático escocês James Gregory (1638-1675) também chegou à noção das séries de Taylor em 1671, quatorze anos antes que Taylor tivesse nascido! Tendo trabalhado em isolamento nos últimos sete anos da sua curta vida e evitado a publicação da sua obra, custou um longo tempo até que o trabalho de Gregory recebesse o reconhecimento devido. Não tanto quanto para os cientistas indianos, que embora tenham antecipado seus pares europeus nesse assunto em no mínimo 150 anos, somente há poucas décadas tiveram seu mérito reconhecido.

\section{Aproximações de pi por séries numéricas}

Para obter uma tabela com um nível de precisão superior à tabela feita por Ariabata em (1), faz-se necessária uma aproximação mais acurada para $\mathrm{R}$ e, consequentemente, do valor de $\pi$. Madhava chegou a usar valor de $\mathrm{R}=3437^{\prime} 44^{\prime \prime} 43^{\prime \prime \prime} 5$ (3437 minutos 44 segundos e 43 terços, ou a sexagésima parte de um segundo) na construção de uma tabela de senos de 24 valores. Entretanto foi a partir dos trabalhos de Jyestadeva em sua obra Yuktibhasa e Nilakanta no seu livro Tantrasangraha que um estudo de séries de potência para o cálculo do valor de $\pi$ apareceu.

A função $\arctan : \mathbb{R} \rightarrow\left(-\frac{\pi}{2}, \frac{\pi}{2}\right)$ é definida como a função inversa da função tan: $\left(-\frac{\pi}{2}, \frac{\pi}{2}\right) \rightarrow \mathbb{R}$, definida por $\tan (\mathrm{x})=\frac{\operatorname{sen}(\mathrm{x})}{\cos (\mathrm{x})}$. Graças ao Cálculo, hoje sabemos que arctan está intimamente relacionada à função $\frac{1}{1+\mathrm{x}^{2}}$, a primeira sendo a integral dessa última. Conforme veremos a seguir, foi a partir dessa relação (sem conhecê-la de antemão, é claro) que os indianos puderam obter uma série numérica para $\pi$.

Para entender o método, observe a Figura 5. Nela, o quadrado OABC tem lado de medida 1 (uma adaptação ao trabalho de Nilakanta) e o lado AB é dividido em n segmentos congruentes $\mathrm{P}_{0} \mathrm{P}_{1}, \ldots, \mathrm{P}_{\mathrm{n}-1} \mathrm{P}_{\mathrm{n}}$. Os segmentos $\mathrm{EF}$ e $\mathrm{P}_{\mathrm{i}-1} \mathrm{D}$ são perpendiculares a $\mathrm{OP}_{\mathrm{i}}$, tornando os triângulos $\mathrm{OEF}$ e $\mathrm{OP}_{\mathrm{i}-1} \mathrm{D}$ semelhantes. Com isso temos

$$
\frac{\mathrm{EF}}{\mathrm{OE}}=\frac{\mathrm{P}_{\mathrm{i}-1} \mathrm{D}}{\mathrm{OP}_{\mathrm{i}-1}}
$$

Também temos que $\mathrm{P}_{\mathrm{i}-1} \mathrm{D} \perp \mathrm{OP}_{\mathrm{i}}$ por construção, fazendo com que $\mathrm{DP}_{\mathrm{i}-1} \mathrm{P}_{\mathrm{i}}$ e $\mathrm{OAP} \mathrm{P}_{\mathrm{i}}$ também sejam semelhantes. Disso obtemos

$$
\begin{aligned}
& \frac{\mathrm{P}_{\mathrm{i}-1} \mathrm{D}}{\mathrm{OA}}=\frac{\mathrm{P}_{\mathrm{i}-1} \mathrm{P}_{\mathrm{i}}}{\mathrm{OP}_{\mathrm{i}}} \\
& \mathrm{P}_{\mathrm{i}-1} \mathrm{D}=\frac{\mathrm{P}_{\mathrm{i}-1} \mathrm{P}_{\mathrm{i}}}{\mathrm{OP}_{\mathrm{i}}} .
\end{aligned}
$$

Substituindo em (12) conseguimos

$$
\mathrm{EF}=\frac{\mathrm{P}_{\mathrm{i}-1} \mathrm{P}_{\mathrm{i}}}{\mathrm{OP}_{\mathrm{i}-1} \mathrm{OP}_{\mathrm{i}}} \approx \frac{\mathrm{P}_{\mathrm{i}-1} \mathrm{P}_{\mathrm{i}}}{\mathrm{OP}_{\mathrm{i}}^{2}}=\frac{\delta}{1+\mathrm{AP}_{\mathrm{i}}^{2}}=\frac{\delta}{1+\mathrm{i}^{2} \delta^{2}},
$$

${ }^{5}$ [2] pág. 413 


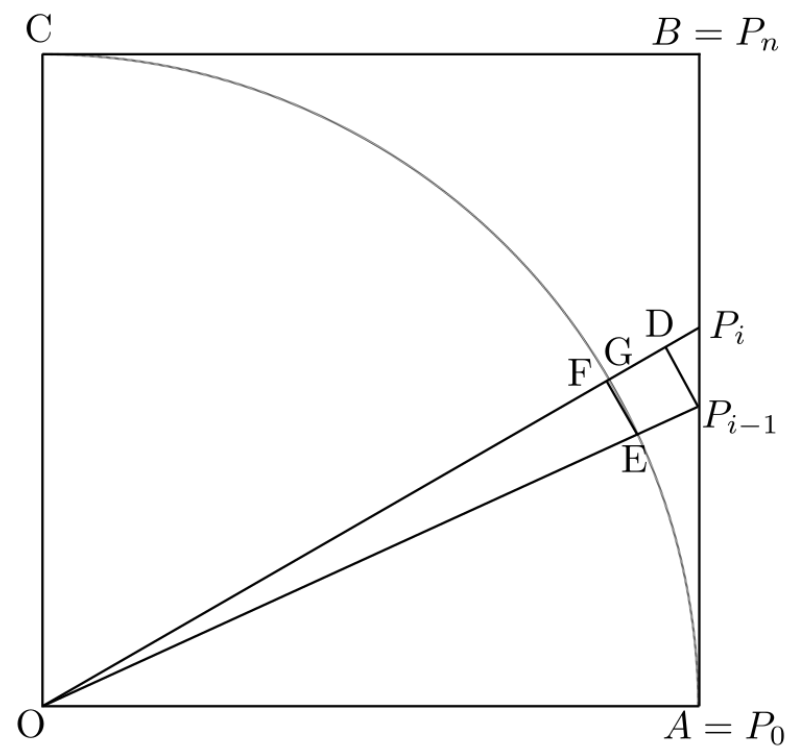

Figura 5: arco de $\frac{1}{4}$ da circunferência em um quadrado de lado 1.

em que $\mathrm{P}_{\mathrm{i}-1} \mathrm{P}_{\mathrm{i}}=\delta=\frac{1}{\mathrm{n}}$, consequentemente, $\mathrm{AP}_{\mathrm{i}}=\mathrm{i} \delta$.

Sendo EG arco da circunferência e usando a aproximação EG $\approx \mathrm{EF}$, temos que

$$
\sum_{\mathrm{i}=1}^{\mathrm{n}} \frac{\delta}{1+\mathrm{i}^{2} \delta^{2}}
$$

é aproximadamente metade do arco AC, correspondendo a $\frac{1}{8}$ do comprimento da circunferência. Como já era conhecido dos indianos que a razão entre o comprimento da circunferência e o diâmetro é constante $(\pi)$, então a expressão acima é igual a $\frac{\pi}{4}$, desde que se fosse possível ter $n$ muito grande. Por mais que as formalidades de limite não estivessem disponíveis para eles, a ideia aqui é preservada, permitindo escrever

$$
\frac{\pi}{4}=\lim _{\mathrm{n} \rightarrow \infty} \sum_{\mathrm{i}=1}^{\mathrm{n}} \frac{\delta}{1+\mathrm{i}^{2} \delta^{2}}=\sum_{\mathrm{i}=1}^{\infty} \frac{\delta}{1+\mathrm{i}^{2} \delta^{2}} .
$$

Para atacar esse problema, Jyestadeva utiliza dois resultados já conhecidos. O primeiro é a soma da série geométrica

$$
\frac{1}{1+x}=1-x+x^{2}-x^{3}+\cdots
$$


que era obtida a partir do seguinte método iterativo

$$
\begin{aligned}
\frac{1}{1+x} & =\frac{1+x-x}{1+x} \\
& =1-x \frac{1}{1+x} \\
& =1-x\left(1-x \frac{1}{1+x}\right) \\
& =1-x+x^{2}\left(1-x \frac{1}{1+x}\right) \cdots
\end{aligned}
$$

O segundo resultado vem de (6), em particular que $\lim _{n \rightarrow \infty} \frac{\sum_{\mathrm{i}=1}^{\mathrm{n}} \mathrm{i}^{\mathrm{k}}}{\mathrm{n}^{\mathrm{k}+1}}=\frac{1}{\mathrm{k}+1}$.

Associando esses dois resultados a (13), temos

$$
\begin{aligned}
\frac{\pi}{4} & =\lim _{n \rightarrow \infty} \sum_{i=1}^{n} \delta-\delta\left(i^{2} \delta^{2}\right)+\delta\left(i^{2} \delta^{2}\right)^{2}-\delta\left(i^{2} \delta^{2}\right)^{3}+\cdots \\
& =\lim _{n \rightarrow \infty}\left(\frac{1}{n} \sum_{i=1}^{n} 1-\frac{1}{n^{3}} \sum_{i=1}^{n} i^{2}+\frac{1}{n^{5}} \sum_{i=1}^{n} i^{4}-\frac{1}{n^{7}} \sum_{i=1}^{n} i^{6}+\cdots\right) \\
& =1-\frac{1}{3}+\frac{1}{5}-\frac{1}{7}+\cdots
\end{aligned}
$$

Essa série de potência é uma conquista. Entretanto, em termos práticos, ela não é muito útil, uma vez que são necessários muitos termos para se obter uma aproximação comparável com as estimativas já obtidas para $\pi$. De fato, para calcular $\pi$ com uma única casa decimal de precisão seriam necessários somar os 300 primeiros termos da série. Para calcular os primeiros 10 dígitos corretamente seriam necessários 5.000.000.000 de termos! Contudo o trabalho de Nilakanta não parou por aí. No livro Tantrasangraha ele expõe métodos que permitem substituir a série original por uma nova série que converge mais rapidamente.

A ideia é a seguinte: considere

$$
\Sigma_{\mathrm{n}}=1-\frac{1}{3}+\frac{1}{5}-\frac{1}{7}+\cdots \pm \frac{1}{\mathrm{n}} \mp \mathrm{f}(\mathrm{n}+1),
$$

em que $f(n)$ é uma fração racional de $n$ tal que $\sum_{n}$ é uma aproximação de $\frac{\pi}{4}$ melhor do que a soma parcial $\mathrm{S}_{\mathrm{n}}=1-\frac{1}{3}+\frac{1}{5}-\frac{1}{7}+\cdots \pm \frac{1}{\mathrm{n}}$. Substituindo $\mathrm{n}$ por $\mathrm{n}-2$ sucessivas vezes em $\sum_{\mathrm{n}}$ temos

$$
\begin{aligned}
\Sigma_{\mathrm{n}-2}= & 1-\frac{1}{3}+\frac{1}{5}-\frac{1}{7}+\cdots \pm \frac{1}{\mathrm{n}-2} \mp \mathrm{f}(\mathrm{n}-1) \\
\Sigma_{\mathrm{n}-4}= & 1-\frac{1}{3}+\frac{1}{5}-\frac{1}{7}+\cdots \mp \frac{1}{\mathrm{n}-4} \pm \mathrm{f}(\mathrm{n}-3) \\
& \vdots \\
\Sigma_{3} & =1-\frac{1}{3}+\mathrm{f}(4) \\
\Sigma_{1} & =1-\mathrm{f}(2) .
\end{aligned}
$$


Agora seja $\mu_{\mathrm{n}}$ definido como

$$
\begin{aligned}
\mu_{\mathrm{n}}= & \Sigma_{\mathrm{n}}-\Sigma_{\mathrm{n}-2}=\frac{1}{\mathrm{n}}-\mathrm{f}(\mathrm{n}+1)-\mathrm{f}(\mathrm{n}-1) \\
\mu_{\mathrm{n}-2}= & \Sigma_{\mathrm{n}-4}-\Sigma_{\mathrm{n}-2}=\frac{1}{\mathrm{n}-2}-\mathrm{f}(\mathrm{n}-1)-\mathrm{f}(\mathrm{n}-3) \\
& \vdots \\
\mu_{3}= & \Sigma_{1}-\Sigma_{3}=\frac{1}{3}-\mathrm{f}(4)-\mathrm{f}(2) .
\end{aligned}
$$

Isso permite reescrever $\sum_{\mathrm{n}}$ como

$$
\begin{aligned}
\Sigma_{\mathrm{n}} & =\Sigma_{1}-\left(\Sigma_{1}-\Sigma_{3}\right)+\left(\Sigma_{5}-\Sigma_{3}\right)+\cdots \mp\left(\Sigma_{\mathrm{n}-4}-\Sigma_{\mathrm{n}-2}\right) \pm\left(\Sigma_{\mathrm{n}}-\Sigma_{\mathrm{n}-2}\right) \\
& =\Sigma_{1}-\mu_{3}+\mu_{5}-\mu_{7}+\cdots \mp \mu_{\mathrm{n}-2} \pm \mu_{\mathrm{n}} \\
& =1-\mathrm{f}(2)-\mu_{3}+\mu_{5}-\mu_{7}+\cdots \mp \mu_{\mathrm{n}-2} \pm \mu_{\mathrm{n}} .
\end{aligned}
$$

Como $\lim _{\mathrm{n} \rightarrow \infty} \Sigma_{\mathrm{n}}=\frac{\pi}{4}$, temos

$$
\frac{\pi}{4}=1-\mathrm{f}(2)-\mu_{3}+\mu_{5}-\mu_{7}+\cdots \mp \mu_{\mathrm{n}-2} \pm \mu_{\mathrm{n}}
$$

que é uma nova série para $\pi$ que depende da escolha de f. Nilakanta propõe então três funções, sem descrever o motivo para tais escolhas ${ }^{6}$ :

$$
\mathrm{f}_{1}(\mathrm{n})=\frac{1}{2 \mathrm{n}}, \mathrm{f}_{2}(\mathrm{n})=\frac{\mathrm{n}}{2\left(\mathrm{n}^{2}+1\right)}, \mathrm{f}_{3}(\mathrm{n})=\frac{\left(\frac{\mathrm{n}}{2}\right)^{2}+1}{\left(\mathrm{n}^{2}+5\right) \frac{\mathrm{n}}{2}} .
$$

Aplicando $f_{1}(\mathrm{n})$ em $\mu_{\mathrm{n}}$ temos

$$
\mu_{\mathrm{n}}=\Sigma_{\mathrm{n}}-\Sigma_{\mathrm{n}-2}=\frac{1}{\mathrm{n}}-\frac{1}{2(\mathrm{n}+1)}-\frac{1}{2(\mathrm{n}-1)}=-\frac{1}{\mathrm{n}^{3}-\mathrm{n}} .
$$

Subsituindo em (14) resulta na série

$$
\frac{\pi}{4}=1-\frac{1}{4}+\frac{1}{3^{3}-3}-\frac{1}{5^{3}-5}+\frac{1}{7^{3}-7}-\cdots
$$

Usando $f_{2}(n)$ vem

$$
\frac{\pi}{4}=\frac{4}{1^{5}+4 \cdot 1}-\frac{4}{3^{5}+4 \cdot 3}+\frac{4}{5^{5}+4 \cdot 5}-\cdots
$$

A função $f_{3}(n)$ é muito eficaz para o cálculo de $\pi$. Sem muita dificuldade, calculando

$$
1-\frac{1}{3}+\frac{1}{5}-\frac{1}{7}+\cdots-\frac{1}{19}+\mathrm{f}_{3}(20)
$$

${ }^{6}$ Pode ser demonstrado que se $\frac{\pi}{4}=\mathrm{S}_{\mathrm{n}}+\mathrm{f}(\mathrm{n}+1)$, então [10]

$$
f(n)=1=\frac{1}{2} \frac{1^{2}}{n+\frac{2^{2}}{n+\frac{3^{2}}{n+\cdots}}}
$$

É praticamente certo que Nilakanta conhecia algum método semelhante ao descrito acima envolvendo frações continuadas para ao cálculo de f, mas o Tantrasangraha não contém nenhuma pista de qual seja ele. 
Nilakanta consegue aproximar $\pi$ com a fração $\frac{104348}{33215}=3,141592654$, correto até a oitava casa decimal. Apesar de não descrito em sua obra como fora a escolha das funções $\mathrm{f}_{\mathrm{i}}$, seus alunos Sankara Variyar [Śañkara Vāriyar] e Narayana [Nārāyaṇa] (c.1500 d.C.) registraram na obra Kriyakramakari uma justificativa matemática para $\mathrm{f}_{1}$ e $\mathrm{f}_{2}$, informando que ela era conhecida por seu mestre $[2$, p. 377].

A aproximação de $\pi$ através da função arctan foi estudada por diversos matemáticos europeus. Em especial, citamos G. W. Leibniz (1673) e J. Gregory (1671), que foram os pioneiros, abordando o problema com motivações distintas, e o matemático suíço Leonhard Euler (1707-1783), que também publicou trabalhos importantes a respeito.

\section{Conclusão}

O grande salto na matemática desenvolvida em Kerala foi a aparição da análise matemática e cálculo numérico na forma de séries e suas aproximações relacionadas a funções no círculo. A motivação para esses trabalhos foi uma curiosidade intelectual, assim como a necessidade de maior precisão em cálculos astronômicos. As demonstrações desses resultados não têm o completo rigor dos padrões atuais, porém são corretas.

As principais descobertas da matemática indiana foram primeiramente relatadas na Europa por Charles Mattew Whish (1794-1833), um funcionário da Companhia das Índias Orientais sediado em Madras (atual Chennai, sudeste da Índia), e durante um século não atraiu a atenção da comunidade científica. O reconhecimento da importância das descobertas indianas veio somente quando o matemático indiano Cadambathur Tiruvenkatacharlu Rajagopal (1903-1978) iniciou uma série de publicações com as descobertas de Whish. Ver mais detalhes em [9] e [10].

Tal "atraso" no reconhecimento dos trabalhos da escola indiana pode estar relacionado à recusa em receber demonstrações fora dos padrões estabelecidos pelo método científico grego e sua correspondente lógica proposicional, além, claro, das limitações em se traduzir os escritos. Esses são tópicos específicos da filosofia da ciência. De acordo com David Pingree em Hellenophilia versus the History of Science [5],

(...) quando a demonstração de Madhava sobre as séries de potência de seno e cosseno foram descritas pela primeira vez em inglês por Charles Whish, na década de 1830, foi alardeado que os indianos descobriram o Cálculo. (...) Essa questão ressurgiu nos anos 50, e agora que temos os textos em sânscrito apropriadamente editados, compreendemos o método engenhoso pelo qual Madhava obteve as séries sem o cálculo; mas muitos historiadores ainda acham impossível a concepção do problema e a sua solução com qualquer outra coisa senão o Cálculo e, por isso, proclamam que foi ao Cálculo que Madhava chegou. Dessa forma a elegância e o brilhantismo da matemática de Madhava são distorcidos na medida em que são soterrados sob a solução matemática corrente de um problema para o qual ele encontrou uma solução alternativa e poderosa.

Essa "competição" velada em se determinar o melhor método - e com isso o único - para obter séries de potência, remete a outro episódio bem conhecido da história do Cálculo: a disputa entre Newton e Leibniz (ou melhor, entre seus admiradores; ou melhor ainda, entre Inglaterra e Alemanha), que trouxeram abordagens diferentes para os mesmos resultados. Em tais batalhas não existem vencedores e hoje reconhecemos que os dois grandes cientistas chegaram a seus resultados 
independentemente e que suas contribuições para o avanço da ciência foram enormes. Com o presente trabalho não esperamos trazer à tona discussão sobre prioridades, mas fazer justiça histórica a um grupo de cientistas que, na Índia, enquanto a Europa aprofundava e despertava do seu sono dogmático da Idade Média, foi capaz de encontrar métodos poderosos para resolver complexos problemas matemáticos.

\section{Referências}

[1] Clark, W.E. The Ariabhatiya of Ariabata. Translated with notes. University of Chicago, 1980.

[2] Joseph, George G. Indian Mathematics: Engaging with the World from Ancient to Modern Times. World Scientific Publishing (UK). 2016.

[3] Katz, V. J. A History of Mathematics: an Introduction, $3^{\text {a }}$ edição, Addison-Wesley, 2009.

[4] Katz, V. J. Ideas of Calculus in Islam and India. Magazine, 68 (1995) pp.163-174.

[5] Pingree, D. E. Hellenophilia versus the History of Science, The University of Chicago Press Journal 562 (1992) Isis 83:4, 554-563.

[6] Plofker, Kim. Mathematics in India. Princeton University Press (2009).

[7] Rodrigues, M. Construções de tabelas de Seno nas civilizações Grega, Árabe e Indiana. Dissertação Profmat, Universidade de Brasília, 2020.

[8] Roy, R. The Discovery of the Series Formula for $\pi$ by Leibniz, Gregory and Nilakantha. Math. Magazine, 63, (1990) pp.291-306.

[9] Rajagopal, C.T. e Rangashari, M.S. On an Untapped Source of Medieval Keralese Mathematics Archive for History of Exact Sciences, vol. 18, 1977, pp.89-102.

[10] Rajagopal, C.T. e Rangashari, M.S. On Medieval Kerala Mathematics, Archive for History of Exact Sciences, vol. 35, 1986, pp.91-99.

Marcos Rodrigues

Centro de Ensino Fundamental 01 do Lago Norte $<$ marcosgt_rodrigues@hotmail.com>

Nilton Barroso Universidade de Brasília <niltonmoura@unb.br>

Recebido: 10/06/2020

Publicado: 02/09/2020 